Dansk Ultralyddiagnostisk Selskab

\title{
Thoracic ultrasound - new challenges, new horizons
}

Christian B. Laursen ${ }^{1,2}$,

Ole Graumann ${ }^{3,4}$,

Najib M. Rahman ${ }^{5,6}$

${ }^{1}$ Department of Respiratory Medicine, Odense University Hospital, Odense, Denmark

2 Odense Respiratory Research Unit (ODIN), Department of Clinical Research, University of Southern Denmark, Odense, Denmark

${ }^{3}$ Department of Radiology, Odense University Hospital, Odense, Denmark

${ }^{4}$ Research and Innovation Unit of Radiology, Department of Clinical Research, University of Southern Denmark, Odense, Denmark

${ }^{5}$ Oxford Centre for Respiratory Medicine, Oxford University Hospitals NHS Foundation Trust, Oxford, United Kingdom

${ }^{6}$ Oxford Respiratory Trials Unit, Nuffield Department of Medicine, University of Oxford, Oxford, United Kingdom

Within this century, thoracic ultrasound (TUS) has been rapidly evolving from a technique known by few initiated experts to a core skill for many clinical specialities assessing patients with suspected respiratory or cardiac disease. In 2020, the process accelerated even further, due to the urgent need of simple bedside diagnostic tools to help triage, diagnose and monitor patients with acute respiratory failure due to COVID-19 [1]. Apart from this urgent clinical need in 2020 , the process have also been facilitated by the recent years continuously steady increase in TUS research and training. At a societal level, TUS have also been gaining more attention, as exemplified by the European Respiratory Society's publication of an official TUS statement and evidence based training program [2, 3]. Based on the aspects, one may rightfully claim that TUS have finally achieved its rightful place alongside the more traditional forms of clinical ultrasound such as ultrasound of the abdomen, heart, and musculoskeletal system.
The research conducted within the last decades have formed a stable and well established platform for TUS, but many areas within the clinical use of TUS still needs further research to ensure evidence based practice. This is exemplified by many clinical guidelines, which are yet to consider if and how TUS should be used within a given clinical setting. The combination of access to relatively cheap, hand-held ultrasound devices and the COVID-19 pandemic have led to a call for more rapid and extensive implementation of TUS in clinical practice [4]. This will most likely lead to an increase use of TUS for assessing patients with acute respiratory conditions other than COVID-19. This poses a clear risk of everyday clinical use of TUS outpacing evidence based clinical practice guidelines. Clinicians, researchers and writers of guidelines therefore have a clear challenge and obligation within the next few years to ensure that the development of both remain in synchrony. The rapid development and publication of an expert consensus on point-of-care ultrasound for COVID-19 is an example of this being possible [1].

The spectrum of clinical use of TUS is uniquely wide, including non-physicians performing TUS using basic hand-held scanners prehospital and even in space, to highly trained experts performing advanced TUS using expensive high-end scanners in very selected in-hospital patients [5, 6] where the differential diagnosis is perhaps more limited. One of the great advantages of many forms of point-of-care ultrasound is its simplicity in asking clinically relevant dichotomous questions [7]. Use of focused TUS to determine the presence or absence of multiple B-lines as part of the interstitial syndrome is a relevant example in this context. The presence or absence of interstitial syndrome has been described as a means of ruling cardiogenic pulmonary oedema in or

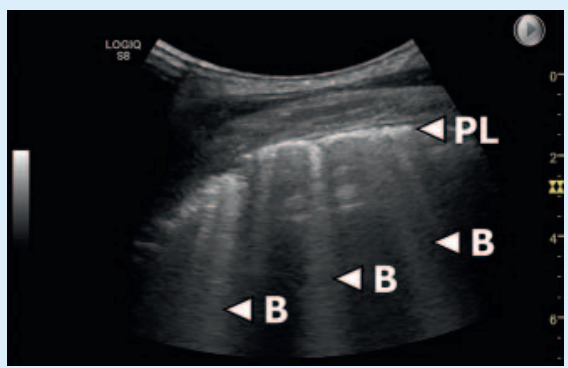

- Fig. 1 TUS image showing a slightly fragmented pleural line (PL) and presence of multiple B-lines. The pattern is clearly abnormal, but does not allow a differentiation between the many causes described as being able to cause such a pattern (e. $\mathrm{g}$. COVID-19, cardiogenic pulmonary oedema, non-cardiogenic pulmonary oedema, interstitial lung disease, near-drowning, lung haemorrhage following transthoracic biopsy) [2]. As with other signs and diagnostic tests, contemplation and careful integration with other findings and clinical context is always warranted.

out in patients with acute respiratory failure [8]. This dogma was further challenged in 2020 as interstitial syndrome is also commonly seen in COVID-19 patients with acute respiratory failure ( $\triangleright$ Fig. $\mathbf{1}$ ). There is no doubt that diagnostic and focused TUS has a rightful place among the standard diagnostic tools used in patients with respiratory symptoms [2]. It is however necessary for any physician using TUS to critically appraise its strengths and limitations, and how presence or absence of different signs and patterns should be understood and integrated in the given clinical context alongside other diagnostic tests performed.

The research in TUS will in future years hopefully continue to push the borders in a wide range of clinical settings. Aspects such as using more advanced techniques as well as using TUS for predicting patient outcomes will undoubtfully be included in the future research $[6,9,10]$. More importantly 
the time has come to agree on standards of TUS quality, methodologies, key outcomes, and how TUS should be integrated in clinical practice guidelines.

\section{References}

[1] Hussain A, Via G, Melniker L et al. Multi-organ point-of-care ultrasound for COVID-19 (PoCUS4COVID): international expert consensus. Critical care 2020; 24: 702

[2] Laursen CB, Clive A, Hallifax R et al. European Respiratory Society Statement on Thoracic Ultrasound. The European respiratory journal: official journal of the European Society for Clinical Respiratory Physiology 2020. doi:10.1183/13993003.01519-2020. Epub ahead of print. PMID: 33033148
[3] ERS Thoracic Ultrasound Certified Training Program. [cited 01.02.2021]; Available from: https://www.ersnet.org/education-andprofessional-development/ers-certifiedtraining-programmes/thoracic-ultrasoundcertified-training-programme/

[4] Farr A, Clementsen P, Herth F et al. Endobronchial ultrasound: launch of an ERS structured training programme. Breathe (Sheff) 2016; 12: 217-220

[5] Kirkpatrick AW, Nicolaou S, Rowan K et al. Thoracic sonography for pneumothorax: The clinical evaluation of an operational space medicine spin-off. Acta Astronautica 2005; 56: 831-838

[6] Jacobsen N, Pietersen PI, Nolsoe C et al. Clinical Applications of Contrast-Enhanced Thoracic Ultrasound (CETUS) Compared to Standard Reference Tests: A Systematic Review. Ultraschall in der Medizin 2020. doi:10.1055/ a-1143-3141. Epub ahead of print. PMID 32259873
[7] Laursen CB, Davidsen JR, Gleeson F. Technique and protocols. In: Laursen CB, Rahman N, Volpicelli G, eds.; Thoracic Ultrasound (ERS Monograph). European Respiratory Society.2018: 14-30

[8] Lichtenstein DA, Meziere GA. Relevance of lung ultrasound in the diagnosis of acute respiratory failure: the BLUE protocol. Chest 2008; 134: 117-125

[9] Sperandeo M, Trovato FM, Dimitri L et al. Lung transthoracic ultrasound elastography imaging and guided biopsies of subpleural cancer: a preliminary report. Acta radiologica (Stockholm, Sweden: 1987) 2015; 56: 798 805

[10] Hassan M, Mercer RM, Rahman NM. Thoracic ultrasound in the modern management of pleural disease. Eur Respir Rev 2020; 29 : 190136. doi:10.1183/16000617.0136-2019 